\title{
Problemi con condizioni omogenee al contorno per una certa classe di operatori formalmente ipoellittici.
}

\author{
Mauro Pagni (a Bologna)
}

Sunto. - Vedere le righe seguenti.

In un precedente lavoro ( $\left.{ }^{1}\right) \mathrm{mi}$ sono occupato dello studio variazionale dei problemi al contorno per una certa classe di operatori quasi-ellittici.

In questa nota tale studio viene esteso prendendo in considerazione una più larga classe di operatori che sono formalmente ipoellittici e più precisamente sono ugualmente forti (nel caso dei coefficienti costanti) di un operatore prodotto di operatori quasi-ellittici $\left({ }^{2}\right)$.

1. - Indichiamo con $x=\left(x_{1}, \ldots, x_{r}\right)$ i punti di $R_{x}^{r}$ (spazio euclideo reale ad $r$ dimensioni), con $y=\left(y_{1}, \ldots, y_{s}\right)$ i punti di $R_{y}^{s}$ (spazio enclideo reale ad $s$ dimensioni) e con $z=(x, y)$ i punti di $R^{m}=R_{x}^{r} \times R_{y}^{s}$.

$$
\begin{gathered}
\text { Poniamo } D_{x_{h}}=-\sqrt{-1} \frac{\partial}{\partial x_{h}}, h=1, \ldots, r, D_{y_{k}}=-\sqrt{-1} \frac{\partial}{\partial y_{k}}, k=1, \ldots, s ; \\
D_{x}^{\alpha}=D_{x_{1}}^{\alpha_{1}} \ldots D_{x_{\psi}}^{\alpha_{r}}, \quad D_{y}^{\beta}=D_{y_{1}}^{\beta_{1}} \ldots D_{y_{s}}^{\beta_{s}} ;|\alpha|=\sum_{i=1}^{r} \alpha_{i},|\beta|=\sum_{j=1}^{s} \beta_{j},
\end{gathered}
$$

$\alpha_{i}, \beta_{i}$ interi non negativi.

Siano $\left\{p_{i}\right\},\left\{q_{i}\right\}(i=1, \ldots, n)$ due $n$-ple di interi positivi assegnati ed indichiamo con I l'insieme delle coppie $(a, b)$, dove $a=\sum_{i=1}^{n} a_{i}, b=\sum_{i=1}^{n} b_{i}$, con $a_{i}, b_{i}$ interi non negativi sodisfacenti alle condizioni $\frac{a_{i}}{p_{i}}+\frac{b_{i}}{q_{i}} \leq 1(i=1, \ldots, n)$. lineare

Detto $\Omega$ un aperto limitato di $R^{m}$ consideriamo l'operatore differenziale

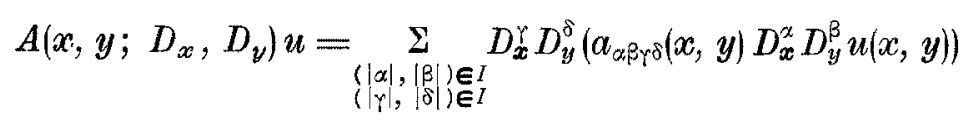

(1) M. PAGNI [1].

(2) Per questioni rignardanti operatori siffatii (ugualmente forti di un prodotto di operatori quasi-ellitici) si veda B. PINI [1], G.O. Barozzi [1], A. OAvaliuocr [1], M. PaGNI $[2],[3]$. 
con $a_{\alpha \beta \gamma \delta}(x, y)$ funzioni complesse limitate e misurabili in $\Omega$ e dove le derivate sono intese nel senso delle distribuzioni in $Q$. L'operatore $A$ viene quindi determinato, fissando le due $n$-ple $\left\{p_{i}\right\},\left\{q_{i}\right\}$ e i coefficienti $a_{\alpha \beta \gamma \delta}(x, y)$ in $\Omega$.

Posto

$$
\begin{aligned}
& K(\zeta)=K(\xi, \eta)=\sum_{(|a|,|\beta|) \in I} \xi^{2 \pi} \eta^{23}, \\
& \xi=\left(\xi_{1}, \ldots, \xi_{r}\right), \quad \eta=\left(\eta_{1}, \ldots, \eta_{s}\right) .
\end{aligned}
$$

indichiamo con

$H_{K}(\Omega)$ lo spazio hilbertiano delle distribuzioni $u$ tali che $D_{x}^{\alpha} D_{y}^{\beta} u \in L^{2}(\Omega)$ per $(|\alpha|,|\beta|) \in I$ con la norma

$$
\|\boldsymbol{u}\|_{H_{K}(\Omega)}=\left(\sum_{(|\alpha|,|\beta|) \in I}\left\|D_{x}^{\alpha} D_{y}^{\beta} u\right\|_{L^{2}(\Omega)}^{2}\right)^{\frac{1}{2}}
$$

$\stackrel{\circ}{H}_{K}(\mathbf{\Omega})$ il sottospazio di $H_{K}(\mathbf{\Omega})$ chiusura di $\mathscr{D}(\mathbf{\Omega})\left({ }^{3}\right)$ in $H_{K}(\mathbf{\Omega})$.

Poichè $\stackrel{\circ}{H}_{K}(\Omega)$ è uno spazio normale di distribnzioni su $\Omega$, il suo duale $\left(\stackrel{\circ}{H}_{K}(\Omega)\right)^{\prime}$ è uno spazio di distribuzioni su $\Omega$. Porremo allora per definizione

$$
H_{K^{-}-(}(\Omega)=\left(\stackrel{\circ}{H}_{K}(\Omega)\right)^{\prime}
$$

$\grave{\mathrm{E}}$ facile provare $\left({ }^{4}\right)$ che lo spazio $H_{K^{-1}}(\Omega)$ è costituito da tutte e sole le distribuzioni $u$ tali che $u=\underset{(|\alpha|,|\beta|) \in I}{\Sigma} D_{x}^{\alpha} D_{y}^{\beta} f_{\alpha \beta} \operatorname{con} f_{\alpha \beta} \in L^{2}(\Omega)$.

2. - Lo studio dei problemi al contorno omogenei per l'equazione

$$
A u=f \text { in } \Omega
$$

si può fare (come vedremo) seguendo l'impostazione generalizzata di J.L. Lions [1], prendendo come "operatori elementari le derivate $D_{x}^{\alpha} D_{y}^{\beta}$ con $(|\alpha|,|\beta|) \in I$. Incominciamo con l'osservare che, per l'ipotesi fatte sui coefficienti, $A u$ può pensarsi definito in $H_{K}(\Omega)$ e non solo $A u \in \mathscr{D}^{\prime}(\Omega)\left({ }^{5}\right)$ ma per quanto sopra detto $A u \in H_{K-1}(\mathbf{Q})$.

( $\left.{ }^{3}\right)$ Spazio delle funzioni complesse $u(x, y)$ indefinitamente differenziabili e a supporto compatto $\subset \Omega$.

(4) Per esempio con ragionamento analogo a quello fatto in E. Magenes e G. StaMPacchia [1] per la dimostrazione del teor. 2.1.

(5) Spazio delle distribuzioni su $\Omega$. 
Associamo ad $A u$ la forma sesquilineare e continua su $H_{K}(\Omega) \times H_{K}(\Omega)$

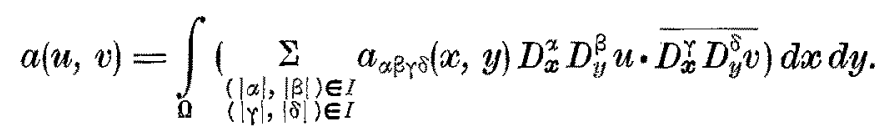

Assegnamo poi :

1) un sottospazio $V_{K}$ (chiuso) di $H_{K}(\Omega)$ tale che $\stackrel{\circ}{H}_{K}(\Omega) \subset V_{K} \subset H_{K}(\Omega)$;

2) uno spazio normale di distribuzioni $\mathcal{Q}$, che sia un BANACH, tale che $v_{K} \subset Q$.

Il duale di $\mathcal{Q}$, ohe indichiamo con $\mathcal{Q}^{\prime}$, è allora uno spazio di distribuzioni in $\Omega$ e riesce $\mathcal{Q}^{\prime} \subset H_{K^{-1}}(\Omega)$.

Denotato con $\mathcal{H}$ lo spazio (di BANACH) della $u \in V_{K}$ per cui $A u \in \mathcal{Q}^{\prime}$, con la norma $\|u\|_{\mathscr{H}}=\left(\|u\|_{V_{K}}^{2}+\|A u\|_{Q^{\prime}}^{2}\right)^{\frac{1}{2}}$, sia infine $\mathscr{O}$ il sottospazio chinso $\mathrm{di}$ $\mathcal{H}$ delle $u$ tali che $\langle A u, \bar{v}\rangle=a(u, v)\left({ }^{\theta}\right)$ per ogni $v \in V_{K}$.

Ciò posto è immediato verificare che l'equazione funzionale nella $u$

$$
a(u, v)=\langle f, \bar{v}\rangle,
$$

per ogni $v \in V_{K}$ e $f$ (fissato) in $\mathcal{Q}^{\prime}$, ̀̀ equivalente al problema al contorno omogeneo

$$
\left\{\begin{array}{l}
A u=f \text { in } \mathbf{Q} \\
u \in \mathscr{O}\left({ }^{\top}\right) .
\end{array}\right.
$$

Nello studio della equazione (2.3) ha un ruolo preponderante la seguente definizione.

DEF. 2.1. - Diremo che la forma $a(u, v)$ è $V_{K}$-ellittica (e che il problema $\grave{e} V_{K}$-ellittico) se esiste una costante $C>0$ tate che

$$
|a(u, u)| \geq C\|u\|_{H_{K}(\Omega)}^{2} \text { per ogni } u \in V_{K}
$$

Sussiste il segnente teorema, la cui dimostrazione si ottiene facilmente facendo ragionamenti analoghi a quelli che si trovano ad es. in E. Magenes e G. Stampacchia [1] e in M. Pagni [1].

TEOR. 2.1. - La $V_{K}$-ellitticit̀̀ di $a(u, v)$ è condizione sufficiente perchè l'equazione (2.3) sia risolubile univocamente.

(6) Con $\left\langle\right.$, $>$ indichiamo la dualità fra $\mathbb{Q}$ e $\mathbb{Q}^{\prime}$.

(7) $u \in \mathscr{O C}$ significa che $u$ soddisfa in senso generalizzato a certe condizioni omogeneo al contorno di 2 . 
3. - Si pone ora il problema di dare delle condizioni di carattere algebrico per la $V_{K}$-ellitticità della forma $a(u, v)$ o per lo meno della forma $a(u, v)+\lambda(u, v)_{L^{2}(\Omega)}\left({ }^{8}\right)$, cioè, come si dice, della «coercività 》 della forma $a(u, v)$.

Ci occuperemo in quel che segue del caso in cui $V_{K}=\stackrel{\circ}{H}_{K}(\Omega)$ (problema di DiRIonLET) ottenendo un risultato che generalizza, agli operatori $A u$ qui considerati, il noto risultato di L. GÅndng per il problema di DIRIoHLeT per gli operatori ellittici.

Considerato l'insieme $I$ (che è stato introdotto al $\mathrm{n}$. 1) diremo che la coppia $\left(a_{*}, b_{*}\right) \in I$ è una coppia massima di $I$ se non esiste alcuna coppia $(a, b) \in I$ tale che $a \geq a_{*}, b \geq b_{*}, a+b>a_{*}+b_{*}$. Indichiamo poi con $I_{*}$ l'insieme delle coppie massime di $I$.

Posto

$$
\|u\|_{* *}=\left(\sum_{(|\alpha|,|\beta|) \in I_{*}}\left\|D_{x}^{\alpha} D_{y}^{\beta} u\right\|_{L^{2}(\Omega)}^{2}\right)^{\frac{1}{2}}
$$

vale la seguente proposizione

Prop. 3.1. - In $\stackrel{\circ}{H}_{K}(\Omega)$ le norme $\|u\|_{*},\|u\|_{H_{K}(\Omega)}$ sono equivalenti.

Dalle definizioni segue che

$$
\|\boldsymbol{u}\|_{*} \leq\|u\|_{H_{K}(\Omega)} \quad \text { per ogni } \quad u \in \stackrel{\circ}{H}_{K}(\Omega)
$$

Per mostrare l'esistenza di una costante $O$ tale che

$$
\|u\|_{H_{K}(\Omega)} \leq C\|u\|_{*} \text { per ogni } u \in \stackrel{\circ}{H}_{K}(\Omega)
$$

incominciamo col provare che, per ogni coppia di multi-indici $(\gamma, \delta)$, esiste una costante $C_{1}(\gamma, \delta)$ tale che per ogni $u \in \mathfrak{D}(\mathbf{\Omega})$ si ha

$$
\left\|D_{x}^{\alpha} D_{y}^{\beta} u\right\|_{L^{2}(\Omega)} \leq C_{1}(\gamma, \delta)\left\|D_{x}^{\gamma} D_{y}^{\delta}\left(D_{x}^{\alpha} D_{y}^{\beta} u\right)\right\|_{L^{2}(\Omega)} .
$$

Infatti se $u(x, y) \in \mathscr{D}(\mathbf{Q})$ riesce

$$
\begin{gathered}
u(x, y)=\int_{-\infty}^{x_{h}} \frac{\partial u}{\partial x_{h}}\left(x_{1}, \ldots, t, \ldots, x_{r} ; y\right) d t \\
\left|\int_{-\infty}^{x_{h}} \frac{\partial u}{\partial x_{h}}\left(x_{1}, \ldots, t, \ldots, x_{r} ; y\right) d t\right|^{2} \leq|\Omega| \int_{-\infty}^{x_{h}}\left|\frac{\partial u}{\partial x_{h}}\right|^{2} d t \leq|\Omega| \int_{-\infty}^{+\infty}\left|\frac{\partial u}{\partial x_{h}}\right|^{2} d t
\end{gathered}
$$

(8) Con $(u, v)_{L^{2}(\Omega)}$ si è indicato il prodotto scalare di $u, v$ in $L^{2(\Omega)}$. 
$(|\Omega|=$ diametro di $\Omega)$; ed integrando su $\Omega$

$$
\int_{\Omega}|u(x, y)|^{2} d x d y \leq|\Omega|^{2} \int_{\Omega}\left|\frac{\partial u}{\partial x_{h}}\right|^{2} d x d y .
$$

Da ciò segue in modo ovvio la (3.3); e da quest'nltima segue la (3.2) per ogni $u \in \mathscr{D}(\Omega)$. Tenuto conto della definizione di $\stackrel{\circ}{H}_{K}(\Omega)$ si ha infine la piena validità della (3.2).

Prop. 3.2. - Ad ogni $\varepsilon>0$ corrisponde un numero $\lambda(\varepsilon)$ tale che

$$
\left(\sum_{(|\alpha|,|\beta|) \in I-I_{*}}\left\|D_{x}^{\alpha} D_{y}^{\beta} u\right\|_{i}^{2}\right)^{\frac{1}{2}} \leq \varepsilon\|u\|_{H_{K}(\Omega)}+\lambda(\varepsilon)\|u\|_{L^{2}(\Omega)}
$$

per ogni $u \in \stackrel{\circ}{H}_{K}(\mathbf{Q})$.

Posto $\xi=\xi_{1}, \ldots, \xi_{r} \in R_{x}^{r}, \eta=\eta_{1}, \ldots, \eta_{s} \in R_{y}^{s}, \zeta=(\xi, \eta) \in R^{m}$

$$
K_{1}(\zeta)=\sum_{(|x|,|\beta|) \in I-I_{*}}^{\Sigma} \xi^{2 x} \eta^{2 \beta}, \quad K_{*}(\zeta)=\sum_{(|\alpha|,|\beta|) \in I_{*}}^{\Sigma} \xi^{2 x} \eta^{2 \beta}
$$

si ha

$$
\frac{K_{1}(\zeta)}{K_{*}(\zeta)+t} \rightarrow 0 \text { per } t \rightarrow 0+\text { uniformemente rispetto a } \zeta \in R^{m}
$$

Infatti osservato che $\lim _{|\zeta| \rightarrow \infty} \frac{K_{1}(\zeta)}{K_{*}(\zeta)+t}=0$, ad ogni $\varepsilon>0$ resta associato un $\rho_{\varepsilon}>0$ tale che per $|\zeta| \geq \rho_{\varepsilon}$ riesce $\frac{K_{1}(\zeta)}{K_{*}(\zeta)+t}<\varepsilon$. Indicato con $M$ il sup di $K_{1}(\zeta)$ nella sfera $|\zeta| \leq \rho_{\varepsilon}$ si ha

$$
\frac{K_{1}(\zeta)}{K_{*}(\zeta)+t}\left\{\begin{array}{llll}
<\varepsilon & \text { per } t>0 & \text { e } & |\zeta| \geq \rho_{\varepsilon} \\
<\frac{M}{t} & \text { per } t>0 & \text { e } & |\zeta| \leq \rho_{\varepsilon}
\end{array}\right.
$$

e quindi per $t>\frac{M}{\varepsilon}, \frac{K_{1}(\zeta)}{K_{*}(\zeta)+t}<\varepsilon$ qualunque $\zeta \in R^{m}$

Infine indicata con $\hat{u}(\zeta)$ la trasformata di FourIER della $u(z)=u(x, y)$,

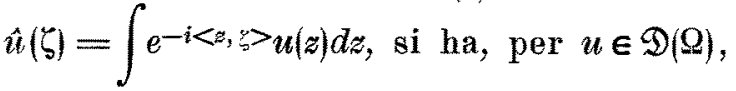

$$
\begin{gathered}
\sum_{(|\alpha|,|\beta|) \in I-I_{*}}^{\Sigma}\left\|D_{x}^{\alpha} D_{y}^{\beta} u\right\|_{L^{2}(\Omega)}^{2}=(2 \pi)^{-m} \int K_{1}(\zeta)|\hat{u}(\zeta)|^{2} d \zeta \\
\sum_{(|\alpha|,|\beta|) \in I_{*}}\left\|D_{x}^{\alpha} D_{y}^{\beta} u\right\|_{L^{2}(\Omega)}^{2}=(2 \pi)^{-m} \int K_{*}(\zeta)|\hat{u}(\zeta)|^{2} d \zeta .
\end{gathered}
$$


Da qui, tenendo conto della (3.5), segue facilmente la voluta proposizione.

DEF. 3.1. - Diremo che l'operatore A soddisfa 'la condizione i) se esiste una costante $c>0 \cdot$ tale che

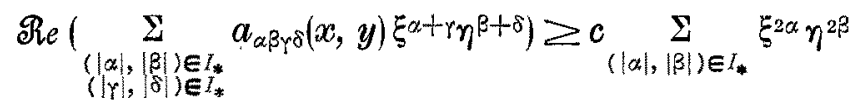

per ogni $(x, y) \in \Omega$ e qualunque sia $(\xi, \eta) \in R^{m}$.

TEOR. 3.1. - Se $i$ coefficienti $a_{\alpha \beta \gamma \delta}(x, y)$ di A, per cui $(|\alpha|,|\beta|),(|\gamma|,|\delta|) \in I_{*}$, sono continui in $\bar{\Omega}$ e se A soddisfa la condizione $i)$, la forma $a(u, v)+\lambda(u, v)_{L^{g}(\Omega)}$ $\grave{e} \stackrel{\circ}{H}_{K}$-ellittica, per $\lambda$ sufficientemente grande.

La dimostrazione di questo teorema, dopo le premesse fatte, si può condurre al solito modo. Posto

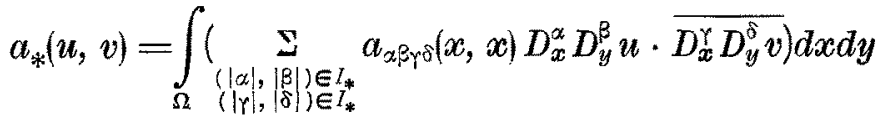

e supposto i coefficienti $a_{\alpha \beta \gamma \delta}$ costanti e che valga la (3.6), si ha

$$
\mathscr{R} e a_{*}(u, u) \geq C\|u\|_{H_{K}(\Omega)}^{2} \text { per ogni } u \in \ddot{H}_{K}(\Omega) \text {. }
$$

Infatti, essendo in $\check{H}_{K}(\mathbf{O})$ le norme $\|u\|_{H_{K}(\Omega)}$ e $\|u\|_{*}$ equivalenti (Prop. 3.1), la (3.7) equivale alla disuguaglianza

$$
\mathfrak{R} e a_{*}(u, u) \geq C_{1}\|u\|_{*}^{2} \text { per ogni } u \in \stackrel{\circ}{H}_{K}(\Omega) \text { (con } C_{1} \text { costante). }
$$

D'altronde condizione necessaria e sufficiente perchè valga la (3.8) per ogni $u \in \stackrel{\circ}{H}_{K}(\Omega)$ è che valga per ogni $u \in \mathscr{D}(\Omega)$,

Orbene per la relazione di Parseval, se $u \in \mathscr{D}(\Omega)$ si ha

$$
\mathscr{R} e a_{*}(u, u)=(2 \pi)^{-m} \int \mathfrak{R e}\left(\underset{\substack{\left.(|\alpha|,|\beta|) \in I_{*} \\|\gamma|,|\delta|\right) \in I_{*}}}{\Sigma} a_{* \beta \gamma \gamma} \xi^{\alpha+\gamma} \eta^{\beta+\delta}\right)|\hat{u}(\zeta)|^{2} d \zeta
$$

e

$$
\left.\|\boldsymbol{u}\|_{*}^{2}=(2 \pi)^{-m} \int_{(|\boldsymbol{x}|,|\mathbb{|}|) \in I_{*}} \xi^{2 \alpha \eta} \eta^{2 \beta}\right)|\hat{u}(\zeta)|^{2} d \zeta
$$

Di qui la (3.8) e quindi la (3.7).

Dopo di ciò, abbandonata l'ipotesi degli $a_{\alpha \beta \gamma \delta}(x, y)$ costanti, si scompone l'integrale

$$
\mathscr{R} e \int_{\Omega}\left(\sum_{\substack{(|\alpha|,|\beta|) \in I \\(|\gamma|,|\delta| \in I}} a_{\alpha \beta \gamma \delta}(x, y) D_{x}^{\alpha} D_{y}^{\beta} u \cdot \overline{D_{x}^{\gamma} D_{y}^{\delta} u}\right) d x d y
$$


mediante partizione dell'unita, in integrali estesi a regioni sufficientemente piccole, e a questi integrali si applica il cosidetto ártificio di KonN riportandosi allora al caso dei coefficienti costanti e si ottiene un $\lambda_{0}$ (servendosi della Prop. 3.2) tale che per $\lambda \geq \lambda_{0}$ riesca

$$
\mathscr{R} e \quad a(u, u) \geq c\|u\|_{H_{K}(\Omega)}^{2}-\lambda\|u\|_{L^{2}(\Omega)}^{2} \quad \text { per ogni } \quad u \in \stackrel{\circ}{H}_{K}(\Omega)
$$

con $c$ costante positiva.

Osservazione - Se $p=p_{1}=\ldots=p_{n}, q=q_{1}=\ldots=q_{n}$ si riottiene come caso particolare il teor. 2.3 di M. PAGNI [1], se di più $p=q$ si riottiene la nota disuguaglianza di L. GÅding per il problema di DiRICHLE⿰亻 per le equazioni ellittiche.

4. - Studieremo in questo n. i legami che intercorrono fra la $V_{K}$-ellitticità della forma $a(u, v)$ e l'ipoellitticità dell'operatore $A$.

Facciamo alcune premesse

Prop. 4.1. - Siano $\Omega$ un aperto limitato $\neq \varnothing d i R^{m}$ e $k_{1}(\zeta), k_{2}(\zeta)$ due funzioni peso temperate $\left(^{9}\right)$ definite in $R^{m}$ per le quali riesca

$$
\int\left|k_{2}(\zeta) \hat{u}(\zeta)\right|^{2} d \zeta C_{1} \int\left|k_{1}(\zeta) \hat{u}(\zeta)\right|^{2} d \zeta \text { per ogni } u \in \mathscr{D}(\Omega)
$$

allora esiste una costante $C$ per cui

$$
k_{2}(\zeta) \leq C k_{1}(\zeta) \quad \zeta \in R^{m}\left({ }^{10}\right) .
$$

Fissiamo una funzione $u(z) \in \mathfrak{D}(\Omega)$ e $\neq 0$ e poniamo $u_{\nu}(z)=u(z) e^{i<z, v>}$. Riesce $u_{y}(z) \in \mathfrak{D}(\Omega)$ e $\hat{u}_{\nu}(\zeta)=\hat{u}(\zeta-v)$. Applicando la (4.1) si ha

$$
\int\left|k_{2}(\zeta+v) \hat{u}(\zeta)\right|^{2} d \zeta \leq C_{1} \int\left|k_{1}(\zeta+v) \hat{u}(\zeta)\right|^{2} d \zeta
$$

Indicata con $M_{k_{i}}(\zeta)(i=1,2)$ la funzione submoltiplicativa associata a $k_{i}$ si ha $\left({ }^{11}\right)$

$$
\begin{aligned}
& k_{1}(\zeta+v) \leq M_{k_{1}}(\zeta) k_{1}(v), \\
& k_{2}(\zeta+v) \geq k_{2}(v) M_{k_{2}}^{-1}(-\zeta), \quad \zeta, v \in R^{m}
\end{aligned}
$$

(9) Secondo la definizione di L. Hörmanoer (L. Hörmander [1] Def, 2.1.1) e cioè una funzione positiva $k(\zeta)$ definita in $R^{m}$ si. chiamerà funzione peso temperata se esistono due costanti positive $C$ e $N$ tali che $k(\zeta+v) \leq(1+C|\zeta|) N k(v) ; \zeta, v \in R^{m}$.

(10) La proposizione enunciata è sostanzialmente contenuta nel Teorema 222 di L. Hörmander [1].

(11) Vedasi L. Hömandfr [1] in quel che segue la Def. 2.1.1. 
e quindi dalla (4.3) la

$$
k_{2}^{2}(\nu) \int\left|M_{k_{2}}^{-2}(-\zeta) \hat{u}(\zeta)\right|^{2} d \zeta \leq C_{1} k_{1}^{2}(v) \int\left|M_{k_{1}}(\zeta) \hat{v}(\zeta)\right|^{2} d \zeta
$$

per ogni $\vee \in R^{m}$. Dalla (4.4) segue la (4.2).

Detto $P(\zeta)$ un polinomio a coefficienti complessi nelle $m$ variabili $\zeta_{1}, \ldots, \zeta_{m}$ poniamo

$$
P(\mu)(\zeta)=\frac{\partial|\mu|}{\partial(\zeta)} \quad \text { e } \quad \tilde{P}(\zeta)^{2}=\Sigma_{|\mu| \geq 0}\left|P^{(\mu)} \ldots \partial \zeta_{m}^{\mu_{m}}(\zeta)\right|^{2}
$$

$\mu_{1}, \ldots, \mu_{m}$ interi non negativi.

Consideriamo i due polinomi

$$
K(\zeta), \text { definito in }(1.2) \text {, e } K_{0}(\zeta)=\prod_{i=1}^{n}\left(\underset{\frac{\left|\alpha^{(i)}\right|}{p_{i}}+\frac{\left|\beta^{(i)}\right|}{q_{i}} \leq 1}{\left.\xi^{2 \alpha^{(i)}} \eta^{2 \beta^{(i)}}\right)}\right.
$$

$\zeta=(\xi, \eta), \alpha^{(i)}=\alpha_{1}^{(i)} \ldots, \alpha_{r}^{(i)}, \beta^{(i)}=\beta_{1}^{(i)} \ldots, \beta_{s}^{(i)}$, con $\alpha_{h}^{(i)}, \beta_{k}^{(i)}$ interi non negativi.

È facile vedere che in essi figurano gli stessi monomi e che quindi (i termini di $K_{0}$ e $K$ sono tutti non negativi) dette $C_{1}$ e $C_{2}$ due costanti opportune si ha

$$
C_{1} K_{0}(\zeta) \leq K(\zeta) \leq C_{2} K_{0}(\zeta), \quad \zeta \in R^{m}
$$

Osservato che $K_{0}(\zeta)=\left|K_{0}(\zeta)\right| \leq \tilde{K}_{0}(\zeta)$, dalla $(4.7)$ segue facilmente che $K(\zeta)$ è ugaalmente forte di $K_{0}(\zeta)\left({ }^{12}\right)$.

D'altronde il polinomio $K_{0}(\zeta)$, prodotto di polinomi quasi-ellittici (tali sono evidentemente i suoi fattori), è ipoellittico $\left({ }^{13}\right)$. Segue allora, da quanto sopra detto, che $K(\zeta)$ è ipoellittico $\left({ }^{14}\right)$.

Supponiamo che la forma $a(u, v)$ associata all'operatore $A=A\left(x, y, D_{x}, D_{y}\right)$ (definito in 1.1) sia $V_{K}$-ellittica e che inoltre $A$ sia a coefficienti costanti $A=A\left(D_{x}, D_{y}\right)=A(D)$.

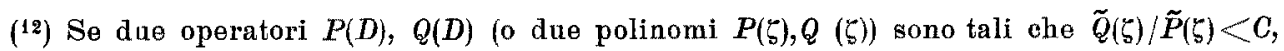
$\zeta \in R^{m}(C$ costante) diremo che $Q$ è più debole di $P$ e scriveremo $Q \prec P$, o che $P$ è più forte di $Q$ e seriveremo $P \prec Q$. Se $P \prec Q \prec P$ i due polinomi verranno detti ugualmente forti. Condizione necessaria e sufficiente perchè $Q \prec P$ è che esista una costante $C_{1}$ tale che $|Q(\zeta)| \leq C_{1} \tilde{P}(\zeta), \zeta \in R^{m}$ (v. L Hörmander [1] Teor. 3.3.2).

(13) Vedasi G.C. Barozzi [1].

(14) Polinomi ugualmente forti di un polinomio ipoellittico sono ipoellittici (v. ad es. Teor. 4.1.6 L. Hörmander [1]). 
In queste ipotesi proveremo che $A(D)$ è ipoellittico: più precisamente che, posto $K(D)=K\left(D_{x}, D_{y}\right)=\underset{(|\alpha|,|\beta|) \in I}{\Sigma} D_{x}^{2 x} D_{y}^{2 \beta}, A(D) \grave{e}$ ugualmente forte di $K(D)$.

Si ha infatti, nelle dette ipotesi, usando la trasformata di Fourier

$$
\left.\left.\left|\int A(\zeta)\right| \hat{u}(\zeta)\right|^{2} d \zeta\left|\geq C \int K(\zeta)\right| \hat{u}(\zeta)\right|^{2} d \zeta \quad \text { per ogni } u \in \mathfrak{D}(\mathbf{\Omega})
$$

D'altronde dall'essere $K(\zeta)$ ipoellitico $e \geq 1$ segue l'esistenza di una costante $C_{1}$ tale che $C_{1} K(\zeta)>\tilde{K}(\zeta)$ per $\zeta \in R^{m}$.

Da ciò, tenuto conto che $\tilde{A}(\zeta) \geq|A(\zeta)|$, si ottiene dalla (48) la

$$
\left.\int\left|\tilde{A}(\zeta)^{\frac{1}{2}} \hat{u}(\zeta)^{2} d \zeta \geq C_{2} \int\right| \tilde{K}(\zeta)^{\frac{1}{2}} \hat{u}(\zeta)\right|^{2} d \zeta \text { per ogni } u \in \mathscr{D}(\Omega)
$$

$\left(C_{2}\right.$ costante positiva).

Ricordato che $\tilde{A}(\zeta), \tilde{K}(\zeta)$ sono due funzioni peso temperate (v. L. HöRMANDER [1]) e che quindi tali sono pure le funzioni $\tilde{A}(\zeta)^{\frac{1}{2}}, \tilde{K}(\zeta)^{\frac{1}{2}}$ (v. L. HöRMander [1] Teor. 2.1.1), per la Prop. 4.1 si ottiene dalla $(4,9)$ che $\tilde{A}(\zeta)>C \tilde{K}(\zeta)$; $\operatorname{cioஓ} A>K$.

È poi immediato verificare che $|A(\zeta)|<C_{1} K(\zeta)$ e quindi che $A<K$. Si conclude cosi nel modo voluto.

Supponiamo infine (abbandonando l'ipotesi dei coefficienti costanti) che siano continui in $\Omega$ i coefficienti $a_{\alpha \beta \gamma \delta}(x, y)$ (di A) per cui $(|\alpha|,|\beta|),(|\gamma|,|\delta|) \in I_{*}$ e naturalmente che la forma $a(u, v)$ associata ad A sia $V_{K}$-ellitlica. Mostreremo che l'operatore $A\left(x, y ; D_{x}, D_{y}\right)$ è in $\Omega$ di «forza costante» più precisamente che detto $\left(x_{0}, y_{0}\right)$ un arbitrario punto di $\Omega$, l'operatore $A\left(x_{0}, y_{0} ; D_{x}, D_{y}\right)$ غे ugualmente forte di $K(D)$.

Sia $\left(x_{0}, y_{0}\right)=z_{0}$ un arbitrario punto di $\Omega$, poniamo per brevità di scrittura

$$
a^{0}(u, v)=\int_{\Omega}\left(\underset{\substack{|\alpha|,|\beta|) \in I \\\langle|\gamma|, \delta|) \in I}}{\Sigma} a_{\alpha \beta \gamma \delta}\left(x_{0}, y_{0}\right) D_{x}^{\alpha} D_{y}^{\beta} u \cdot \overline{\left.D_{x}^{\gamma} D_{y}^{\delta} v\right)} d x d y\right.
$$

Dalla supposta $V_{K}$-ellitticità di $a(u, v)$ si ha

$$
|\alpha(u, u)| \geq C\|u\|_{H_{K}(\Omega)}^{2} \text { per ogni } u \in \mathfrak{D}(\mathbf{\Omega}) .
$$

Tenuto conto della continuità dei coefficienti $a_{x \beta \gamma \delta}(x, y)$ (quelli per eui $\left.(|\alpha|,|\beta|),(|\gamma|,|\delta|) \in I_{*}\right)$, fissato $\varepsilon$ positivo $e<\frac{C}{4 l}(C$ è la costante che figura

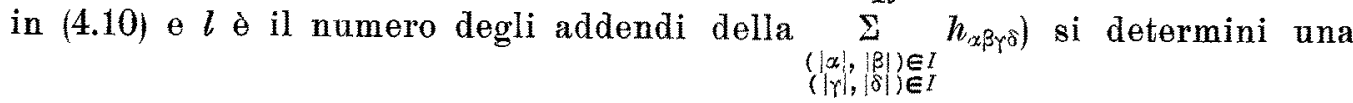


sfera aperta di centro $z_{0}$, contenata in $\boldsymbol{\Omega}$, sfera che indicheremo con $\mathbf{\Omega}_{0}$, tale che

$$
\underset{\substack{\left(|\alpha|,|\beta|,\left|\in I_{*}\\\right|,|,|, \mid \in I_{*}\right.}}{\sum}\left|a_{\alpha \beta \gamma \delta}(x, y)-a_{\alpha \beta \gamma \delta}\left(x_{0}, y_{0}\right)\right|<\varepsilon \text { per ogni }(x, y) \in \Omega_{0} .
$$

Si ha evidentemente

$$
|a(u, u)| \geq C\|u\|_{H_{K}(\Omega)}^{2} \quad \text { per ogni } \quad u \in \mathscr{D}\left(\Omega_{0}\right)
$$

e quindi

(4.13) $\left|a(u, u)-a^{0}(u, u)\right|+\left|a^{0}(u, u)\right| \geq C\|u\|_{H_{K}(\Omega)}^{2} \quad$ per ogni $\quad a \in \mathscr{D}\left(\Omega_{0}\right)$.

D'altronde per $u \in \mathfrak{D}\left(\boldsymbol{\Omega}_{0}\right)$

$\left|a(u, u)-a^{0}(u, u)\right|=\mid \int_{\Omega_{0}}\left(\sum_{\substack{\alpha, \alpha,|\beta| \in I_{*} \\(|\gamma,| \beta \mid) \in I_{*}}}\left[a_{\alpha \beta \gamma \delta}(x, y)-a_{\alpha \beta \gamma \delta}\left(x_{0}, y_{0}\right)\right] D_{x}^{\alpha} D_{y}^{\beta} u \cdot \overline{D_{x}^{\gamma} D_{y}^{\delta} u \mid} d x d y+\right.$

$$
\begin{aligned}
& +\int_{\Omega_{0}} \sum_{\substack{\left.(|\alpha|,|\beta|) \in I-I_{*} \\
|| \gamma|,| \delta \mid\right) \in I-I_{*}}}\left[a_{\alpha \beta \gamma \delta}(x, y)-a_{\alpha \beta \gamma \delta}\left(x_{0}, y_{0}\right)\right] D_{x}^{\alpha} D_{y}^{\beta} u \cdot \overline{\left.D_{x}^{\gamma} D_{y}^{\delta} u\right)} d x d y \mid \leq \\
& \leq l \varepsilon\|u\|_{H_{K}(\Omega)}^{2}+2 l M \underset{(|\alpha|,|\beta|) \in l-l, l_{*}}{\mathrm{\Sigma}}\left\|D_{x}^{\alpha} D_{y}^{\beta} u\right\|_{L^{2}(\Omega)}^{2},
\end{aligned}
$$

dove $M$ è un numero $>\left|a_{a \beta \gamma \gamma \delta}(x, y)\right|$ per $\left(|\alpha|, \mid \beta_{i}\right),(|\gamma|,|\delta|) \in I$ e $(x, y) \in \Omega$.

Per la Prop. 3.2 si potra determinare un $\rho>0$ tale che il secondo addendo dell'ultimo membro di $(4.14)$ sia $<\varepsilon\left\|\left.u\right|_{H_{K}(\Omega)} ^{2}+f\right\| u \|_{L^{2}(\Omega)}^{2}$ per ogni $u \in \mathscr{D}\left(\Omega_{0}\right)$. Si ha cosi dalla (4.14)

$$
\left|a(u, u)-a^{0}(u, u)\right| \leq \frac{C}{2}\|u\|_{H_{K}(\Omega)}^{2}+\odot\|u\|_{L^{2}(\Omega)}^{2} \text { per ogni } u \in \mathfrak{D}\left(\Omega_{0}\right) .
$$

Dalla (4.13) e (4.15) segue

$$
\left|a^{0}(u, u)\right|+\rho\|u\|_{L^{2}(\Omega)}^{2} \geq \frac{C}{2}\left\|u_{i}^{\prime}\right\|_{H^{(}(\Omega)}^{2} \quad \text { per ogni } \quad u \in \mathscr{D}\left(\Omega_{0}\right) .
$$

Di qui usando la relazione di Parseval,

(4.17) $\int\left(\left|A\left(z_{0} ; \zeta\right)\right|+\rho\right)|\hat{u}(\zeta)|^{2} d \zeta \geq \frac{C}{2} \int K(\zeta)|\hat{u}(\zeta)|^{2} d \zeta$ per ogni $u \in \mathscr{D}\left(\mathbf{\Omega}_{0}\right)$ 
Tennto conto di quanto si è detto in precedenza nel caso dei coefficienti costanti si ha

$$
\tilde{A}\left(z_{0} ; \zeta\right)+\rho>C_{1} \tilde{K}(\zeta) \quad \zeta \in R^{m}, \quad C_{1} \text { costante positiva. }
$$

Da qui osservato che $\lim _{|\zeta| \rightarrow \infty} \tilde{K}(\zeta) \rightarrow \infty$, segue

$$
\tilde{A}\left(z_{0} ; \zeta\right) \rightarrow \infty \text { per }|\zeta| \rightarrow \infty \text {. }
$$

Essendo $\tilde{A}\left(z_{0} ; \zeta\right)>0\left(\tilde{A}\left(z_{0} ; \zeta\right)=0\right.$ per qualche $\zeta$, comporta $\left.\tilde{A}\left(z_{0} ; \zeta\right) \equiv 0\right)$ si ha, tenuto conto della (4.19), che esiste una costante $C$ tale che $C \tilde{A}\left(z_{0} ; \zeta\right)>\tilde{A}\left(z_{0} ; \zeta\right)+\rho$. Sị ottiene così, in virtù della $(4.18)$, che $A\left(z_{0} ; \zeta\right)>K(\zeta)$. Essendo, ovviamente, $A\left(z_{0} ; \zeta\right)<K(\zeta)$ si conclude che $A\left(z_{0} ; \zeta\right)$ e $K(\zeta)$ sono egualmente forti.

Quanto detto nel presente n. si compendia nel

TEOR. 4.1. - Dalla $V_{K}$-ellitlicità di $a(u, v)$, nelle ipotesi di continuità in $\Omega$ dei coefficienti $a_{\alpha \beta \gamma \delta}(x, y)$ per cui $(|\alpha|,|\beta|),(|\gamma|,|\delta|) \in I_{*}$, segue che, in ogni punto $\left(x_{0}, y_{0}\right) \in \Omega$, l'operatore $A\left(x_{0}, y_{0}, D_{x} ; D_{y}\right) \grave{e}$ ugualmente forte $d i$ $K\left(D_{\infty}, D_{y}\right)=\underset{(|\alpha|,|\beta|) \in I}{\mathrm{\Sigma}} D_{x}^{2 \alpha} D_{y}^{2 \beta}$.

Il teorema ora enunciato è assai utile per lo studio della regolarizzazione delle soluzioni dei problemi al contorno qui considerati ( $V_{K}$-ellittici) perchè permette di utilizzare $i$ già noti risultati sulla regolarizzanione delle soluzioni delle equazioni ipoellittiche $\left({ }^{15}\right)$.

5. - Terminiamo con un semplice esempio riguardante il problema di DIRICHLET.

Consideriamo l'operatore $A u=\frac{\partial^{8} u}{\partial x^{4} \partial y^{4}}-\frac{\partial^{6} u}{\partial x^{6}}-\frac{\partial^{6} u}{\partial y^{6}}$, che $\partial$ uno dei più semplici operatori ipoellittici di ordine pari non quasi-ellittico.

Volendo interpretare esplicitamente le condizioni al contorno è opportuno considerare l'aperto $\mathrm{Q}=\{(x, y): a<x<b, c<y<d\} \quad\left({ }^{16}\right)$.

Nel caso dell'operatore preso in esame è $p_{1}=q_{2}=2, p_{2}=q_{1}=1$ e l'insieme $I$ è costituito dalle disposizioni, con ripetizione, a due a due dei numeri $0,1,2$ e dalle coppie $(3,0),(0,3) . I_{*}$ (l'insieme delle coppie massime) è costituito dalle coppie $(3,0),(0,3),(2,2) . H_{K}(\mathbf{Q})$ è formato dall'insieme delle funzioni $u(x, y)$ dotate di derivate (nel senso delle distribuzioni in $\Omega$ ) di quadrato sommabile in $\Omega$ sino al terzo ordine e aventi la derivata $\frac{\partial^{4} u}{\partial x^{2} \partial y^{2}}$ pure di

(15) Si veda $L$ Hörmander [1] e quanto detto in [2.4] di M. Pagni [1].

(16) Si è già segnalata ampiamente questa opportunita in M. PAGNI [1]. 
quadrato sommabile in $\Omega$. La norma $\|u\|_{H_{K}(\Omega)}$ è data dalla radice quadrata della somma dei quadrati delle norme in $L^{2}(\Omega)$ di dette derivate.

亡̀ subito visto che, fatto $V_{K}=\stackrel{\circ}{H}_{K}(\Omega)$, il problema è $V_{K}$-ellittico e che quindi il problema di DIRICHLET con dati al contorno omogenei è «ben posto» $\left.{ }^{17}\right)$. Detto problema equivale (prendiamo $Q=\stackrel{\circ}{H}_{K}(\mathrm{Q})$ ) a

$$
\begin{aligned}
& A u=f \text { in } \quad \Omega, f \in H_{K^{-}}(\Omega) \\
& u=0 \quad \text { su } \partial \Omega, \frac{\partial u}{\partial x}=\frac{\partial^{2} u}{\partial x^{2}}=0 \quad \text { sui lati } x=a, x=b, c<y<d, \\
& \frac{\partial u}{\partial y}=\frac{\partial^{2} u}{\partial y^{2}}=0 \text { sui lati } y=c, y=d, a<x<b ;
\end{aligned}
$$

l'annullarsi dei dati al contorno essendo inteso nel senso delle tracce di $u$ come elemento di $H_{\mathrm{K}}(\Omega)\left({ }^{18}\right)$.

Si osservi che pur essendo l'operatore di ottavo ordine i dati del problema di Dirichlet sono solo tre.

\section{BIBLIOGRAFIA}

G.C. BarozzI : [1] Sul prodotto di polinomi quasi-ellittici; Boll. U. M.I. s. III, Anno XX, 1965.

A. Cavallucci: [1] Su una questione di invarianza di polinomi ugualmente forti a prodotti di polinomi quasi-ellittici; Boll. U. M.I. s. III, Anno XX, 1965.

L. HöRMANDER: [1] Linear partial differential operators; Springer, Berlin, 1963.

J. L. Lrons: [1] Problémes aux limites en thérie des distributions; Acta Math. vol. 94, 1950.

E. MaGenes e G. STAMPaCchia: [1] I problemi al contorno per le equazioni differenziali di tipo ellittico; Ann. Se. Normale Sup. Pisa, vol. X1I, 1958.

M. PAGN1: [1] Problemi al contorno per una certa classe di equazioni tineari alle derivate paraiali; Atti Sem. Mat. e Fis. Univ. Moderna, vol. XIII, 1964. [2] Un teorema di tracce; Rend Ace. Naz. Lincei, s. VIII, vol. XXXVIII, 196ó [3] Problemi al contorno per una certa classe di operatori ipoellittici; Atti del Convegno di Nervi sulle equazioni a derivate parziali, 1965, Cremonese, Roma.

B. PINI : [1] Sulle tracee di an certo spaeio funeionale I; Rend. Acc. Naz. Lincei, s. VIII, vol. XXXVII, 1964, II ibidem, vol. XXXVIII, 1965.

(17) Se prendíamo $Q=\stackrel{\circ}{H}_{K}(\Omega)$ si ha che $A u$ è un isomorfismo algebrico e topologico di $\stackrel{\circ}{H}_{K}(\Omega)$ su $H_{K^{-1}}(\Omega)$.

(18) Si veda B. PInI [1] e M. PAgni [2]. 